\title{
Not the end of the road
}

What happens to a dendritic cell (DC) when it has completed its journey from the peripheral tissues to the secondary lymphoid organs, where it presents antigen to naive T cells? Previous in vitro studies have shown that these mature DCs succumb to activationinduced apoptosis. But new work published in Nature Immunology indicates that the path of DC involvement might be longer than previously thought: mature DCs can differentiate further to a regulatory phenotype under the influence of the lymphoid microenvironment.

Zhang et al. cultured mature DCs on monolayers of endothelial-like splenic stromal cells (ESSCs) to mimic the secondary lymphoid microenvironment. These DCs continued to proliferate for several months, whereas mature DCs cultured alone die after $\sim 1$ week. The ESSCs also induced a distinct DC phenotype involving downregulation of expression of MHC class II molecules and increased expression of some co-stimulatory molecules, such as CD40 and CD80. These DCs produced more interleukin-10 (IL-10) and nitric oxide but less transforming growth factor- $\beta$ (TGF- $\beta$ ) and IL-12 than mature DCs.

The induction of mature DC proliferation was shown to require direct cell contact with ESSCs, because fixed ESSCs, but not ESSC culture supernatants, could reproduce the effect. The addition of blocking antibodies specific for fibronectin inhibited ESSC-induced proliferation of mature DCs, indicating one pathway by which this cell contact might be mediated. By contrast, the further differentiation of mature DCs was shown to require both cell contact and ESSC-derived soluble factors. A blocking antibody specific for TGF- $\beta$ inhibited the further differentiation of mature DCs induced by ESSC supernatant, indicating that this is a crucial soluble factor.

Ovalbumin (OVA)-pulsed ESSCdifferentiated DCs did not promote the proliferation of OVA-specific
$\mathrm{CD}^{+} \mathrm{T}$ cells and could inhibit T-cell proliferation induced by OVA-pulsed mature DCs. However, the ESSCdifferentiated DCs did induce T-cell activation, as indicated by cell-surface marker expression and increased production of IL-2 and interferon- $\gamma$. The inhibitory function of these DCs on T-cell proliferation was shown to depend on the production of soluble factors and not on cell-cell contact, and a selective inhibitor of nitricoxide synthase reversed the inhibition of T-cell proliferation. Also, T cells activated by ESSC-differentiated DCs did not function as regulatory cells when added to mature DC and T-cell co-cultures. Taken together, these results indicate that the increased production of nitric oxide by ESSCdifferentiated DCs directly inhibits T-cell proliferation, without the generation of regulatory $\mathrm{T}$ cells that has been shown for other DC subtypes.

Finally, the authors looked for an in vivo counterpart to this new DC phenotype observed in vitro. The main distinguishing characteristic in vitro was decreased expression of MHC class II molecules, so they sorted splenic CD11 $\mathrm{c}^{+}$DCs into negative, low and high populations on the basis of MHC class II expression. The DCs with low-level expression of MHC class II had a similar cell-surface phenotype to ESSC-differentiated DCs and also produced high levels of IL-10 and nitric oxide. Their function was also similar in that they inhibited the proliferation of T cells induced by DCs that expressed high levels of MHC class II.

So, the authors suggest that, rather than being the end of the road for mature DCs, the lymphoid microenvironment can induce a regulatory DC phenotype that might have a role in maintaining immune homeostasis by exerting negative feedback at late stages of a response.

Kirsty Minton

(1) References and links

ORIGINAL RESEARCH PAPER Zhang, M. et al. Splenic stroma drives mature dendritic cells to differentiate into regulatory dendritic cells. Nature Immunol. 5, 1124-1133 (2004).
IN BRIEF

NATURAL KILLER T CELLS

A subset of liver NK T cells is activated during Leishmania donovani infection by CD1d-bound lipophosphoglycan.

Amprey, J. L. et al. J. Exp. Med. 200, 895-904 (2004).

This is the first paper to show that presentation of a pathogenderived glycolipid antigen by CD1d to a subset of natural killer T (NKT) cells has a key role in resistance to Leishmania donovani infection of mice. CD1d-deficient mice have increased susceptibility to various microbial pathogens, and the authors show that they also have increased susceptibility to L. donovani. In wild-type mice, L. donovani infection induces rapid production of interferon- $\gamma($ IFN- $\gamma)$ by CD1d-restricted NKT cells in the liver. In vitro binding assays showed that $L$. donovani lipophosphoglycan (LPG) and other related glycolipids bind strongly to CD1d, and LPG-treated dendritic cells could stimulate IFN- $\gamma$ production by hepatic lymphocytes, indicating that glycolipid-specific responses are likely to contribute to resistance to leishmaniasis.

\section{T CELLS}

Unique gene expression program of human germinal center T helper cells.

Kim, C. H. et al. Blood 104, 1952-1960 (2004).

Germinal-centre T helper (GC- $\mathrm{T}_{\mathrm{H}}$ ) cells are specifically localized in the germinal centres of lymphoid follicles to help $\mathrm{B}$ cells produce antibodies. They are characterized by expression of CXCR5 and CD57, and are non-polarized in terms of cytokine production. Now, Eugene Butcher and colleagues have used cDNA-microarray analysis to find out more about the differences between $\mathrm{GC}-\mathrm{T}_{\mathrm{H}}$ cells and other T-cell subsets. In particular, $\mathrm{GC}-\mathrm{T}_{\mathrm{H}}$ cells are unique in their expression of the chemokine CXCL13, which is crucial for B-cell entry to lymphoid follicles but was previously thought to be produced by non-T cells, such as follicular dendritic cells. They also found marked differences in the expression of transcription factors between $\mathrm{GC}-\mathrm{T}_{\mathrm{H}}$ cells and other T-cell subsets, which supports the theory that GC- $\mathrm{T}_{\mathrm{H}}$ cells are a novel functional lineage.

\section{ASTHMA AND ALLERGY}

Defining a link with asthma in mice congenitally deficient in eosinophils.

Lee, J. J. et al. Science 305, 1773-1776 (2004).

A critical role for eosinophils in allergic airway remodeling. Humbles, A. A. et al. Science 305, 1776-1779 (2004).

Two studies in Science have used different transgenic mice that lack eosinophils to look at how these cells are involved in the pathogenesis of asthma. Both studies reveal an essential role for eosinophils, but they differ in terms of the stage that is affected. Lee et al. show that eosinophils are required for mucus accumulation and airway hyper-responsiveness. By contrast, Humbles et al. show that eosinophils are not required for this acute lung dysfunction but are necessary for the collagen deposition and smooth-muscle hyperplasia that lead to chronic airway remodelling. Regardless of these differences, both studies indicate that eosinophils would be a good therapeutic target for human asthma. 